\title{
CHEMISTRY IN THE EARLY UNIVERSE
}

\author{
PAUL R. SHAPIRO \\ Department of Astronomy \\ The University of Texas at Austin \\ Austin, TX 78712 USA
}

\begin{abstract}
Galaxies and the first stars in the universe formed billions of years ago as a result of the cooperative effects of gravitational collapse and nonequilibrium chemistry. Gravity drew the primordial gas together into lumps; the formation of the first molecules in the universe, simple diatomic molecules like $\mathrm{H}_{2}, \mathrm{H}_{2}^{+}, \mathrm{HD}$, $\mathrm{HeH}^{+}, \mathrm{LiH}$, and $\mathrm{LiH}^{+}$, may then have ensured that the heat generated by gravitational collapse and shock waves was radiated away rapidly enough to allow the gravitational collapse and fragmentation of these gaseous lumps to proceed to the point of forming stars and galaxies. We briefly mention a few of the latest studies of this primordial chemistry, including that in the evolving intergalactic medium (IGM) in a Cold Dark Matter (CDM) model cosmology and that in radiative shocks in the early universe.
\end{abstract}

\section{Introduction}

Molecule formation in the early universe has been studied under three related circumstances: (1) the uniform pregalactic IGM at high redshift $\mathrm{z}$ in the postrecombination epoch $\left(z \lesssim 10^{3}\right)[1-4]$; $(2)$ the gravitational collapse of primordial gas clouds [5-16]; and (3) the radiative shocks which occur in a wide range of galaxy and primordial star formation theories [17-29]. In view of the space limitation here, I will just mention a few highlights of work completed since the last reviews of this subject $[25,30]$ and refer the reader to those reviews for the full background and references.

When the universe recombined at $z \sim 10^{3}$, the gas was composed primarily of $\mathrm{H}, \mathrm{D},{ }^{4} \mathrm{He},{ }^{3} \mathrm{He}$, and ${ }^{7} \mathrm{Li}$, with nuclear abundances by number relative to $\mathrm{H}$ of order $1,5 \times 10^{-5}, 10^{-1}, 10^{-5}$, and $10^{-10}$, respectively [31]. In this essentially metal-free 
gas, $\mathrm{H}_{2}$ was likely to have been the most abundant molecule and was potentially important as a source of radiative cooling in collapsing pregalactic clouds and in shock-heated pregalactic gas. Since $\mathrm{H}_{2}$ has no dipole moment, formation by direct radiative association of two $\mathrm{H}$ atoms is very slow, and $\mathrm{H}_{2}$ formation is thought to have been dominated, instead, by the creation of $\mathrm{H}^{-}$by radiative attachment, followed by associative detachment $[5,6]$ :

$$
\begin{gathered}
H+e^{-} \rightarrow H^{-}+\gamma \\
H+H^{-} \rightarrow H_{2}+e^{-}
\end{gathered}
$$

and by the creation of $\mathrm{H}_{2}^{+}$by radiative association, followed by charge transfer [32]:

$$
\begin{gathered}
H+H^{+} \rightarrow H_{2}^{+}+\gamma \\
H_{2}^{+}+H \rightarrow H_{2}+H^{+}
\end{gathered}
$$

\section{2. $\mathrm{H}_{2}$ in the IGM in a Postrecombination CDM Universe}

Previous studies of molecule formation in the pregalactic IGM beginning with the recombination epoch at $z \cong 10^{3}$ considered a uniform expanding IGM of fixed comoving density $\Omega_{I G M}$ (in units of the critical density for an Einstein-deSitter universe) and focused on the epoch prior to nonlinear structure formation in the universe [1,2]. (An exception is [3], which considered the effect of radiation from postulated primordial stars at high z.) As part of our general study of the IGM, we have reconsidered this problem within the context of the recently popular CDM model for galaxy and large-scale structure formation. We have coupled our detailed, numerical calculations of the thermal and ionization balance, molecule formation, and radiative transfer in a uniform IGM of $\mathrm{H}$ and $\mathrm{He}$ to the linearized equations for the growth of density fluctuations in both the gaseous and dark matter components $[4,33,34]$. The mean IGM density parameter in this case is $\Omega_{I G M}(z)=\Omega_{b}\left[1-f_{c}(z)\right]$, where $\Omega_{b}$ is the total baryon mass fraction in the universe and $f_{c}(z)$ is the timevarying, collapsed baryon fraction. We consider a standard CDM model with $\Omega_{t o t}=1, \Omega_{b}=0.1$, and Hubble constant $h=H_{o} /\left(100 \mathrm{~km} \mathrm{~s}^{-1} \mathrm{Mpc}^{-1}\right)=0.5$, with a power spectrum normalized so that the rms density fluctuation evaluated using a "top-hat" filter is $\sigma_{o}=1 / b$ at $R=8 h^{-1} \mathrm{Mpc}$ at present, where $b$ is the so-called bias parameter. In order to account for the observed absence of a detectable $\mathrm{H} \mathrm{Ly}$ $\alpha$ absorption trough in the spectra of high $\mathrm{z}$ quasars due to a smoothly distributed IGM (so-called Gunn-Peterson effect; "GP"), we assume that the collapsed fraction 
releases just enough ionizing radiation at a constant rate per collapsed baryon, with an AGN-like spectrum $\left(F_{\nu} \propto \nu^{-1.5}\right)$, to reduce the GP optical depth to less than 0.1 by $z=4.1$. This minimal emissivity corresponds to $1200(b=1)$ or 24,400 $(b=2.6)$ ionizing photons per collapsed baryon per present Hubble time.

Shown in Figure 1 are some results of our calculations, which solve rate equations for the concentrations of $\mathrm{H}^{o}, \mathrm{H}^{+}, \mathrm{He}^{o}, \mathrm{He}^{+}, \mathrm{He}^{++}, \mathrm{H}_{2}, \mathrm{H}_{2}^{*}$ (vibrationally excited), $\mathrm{H}_{2}^{+}, \mathrm{H}^{-}$, and $\mathrm{e}^{-}$, the energy conservation equation, including the effects of cosmological expansion, radiative cooling, and cooling (or heating) by Compton scattering of the cosmic microwave background (CMB), and the equation of radiative transfer, including the opacity of the IGM and its own diffuse emission, as well as the mean opacity of an evolving distribution of gas clumps (i.e. quasar absorption-line clouds) embedded in the smoothly distributed IGM. As in previous studies, $\mathrm{H}_{2}$ formation shortly after recombination was limited by the photodestruction of $\mathrm{H}^{-}$and $\mathrm{H}_{2}^{+}$by the CMB. It is important in this case to take account of the population of excited states of $\mathrm{H}_{2}^{+}$with an excitation temperature equal to the CMB radiation temperature. Otherwise, photodissociation of $\mathrm{H}_{2}^{+}$at high $\mathrm{z}$ is underestimated, and $\mathrm{H}_{2}$ formation is spuriously accelerated and enhanced. For 200 $\lesssim z \lesssim 600$, the $\mathrm{H}_{2}^{+}$process dominated the formation of $\mathrm{H}_{2}$, yielding a concentration $\lesssim 10^{-6}$ by $z \sim 300$. By $z \sim 100$, the $\mathrm{H}^{-}$process boosted this to $\sim 2 \times 10^{-6}$, before the collapsed fraction finally released enough radiation by $z \lesssim 25(b=2.6)$ or $z \lesssim 50(b=1)$ to heat and ionize the IGM, destroying the $\mathrm{H}_{2}$. Figure 1 shows that once IGM reionization began, unshielded photodissociation would have destroyed the $\mathrm{H}_{2}$ more quickly than collisional dissociation, but even in the fully self-shielded limit (no photodissociation), collisions would have been more than enough to reduce the $\mathrm{H}_{2}$ concentration by many orders of magnitude to undetectable levels even before the GP constraint was satisfied.

\section{Radiative Shocks and Nonequilibrium $\mathrm{H}_{2}$ Chemistry in the Early Universe}

Shock waves in a metal-free gas are predicted to occur in the IGM and inside protogalaxies under a wide range of circumstances in the theory of galaxy and primordial star formation, including gravitational collapse of density fluctuations, cloud-cloud collisions, and blast waves from the explosive heating of the IGM. In all cases, the radiative cooling of the postshock gas is essential in order to dissipate enough gravitational or explosion energy to make gravitational instability and fragmentation possible in the shock-heated gas, a prerequisite for galaxy or star 


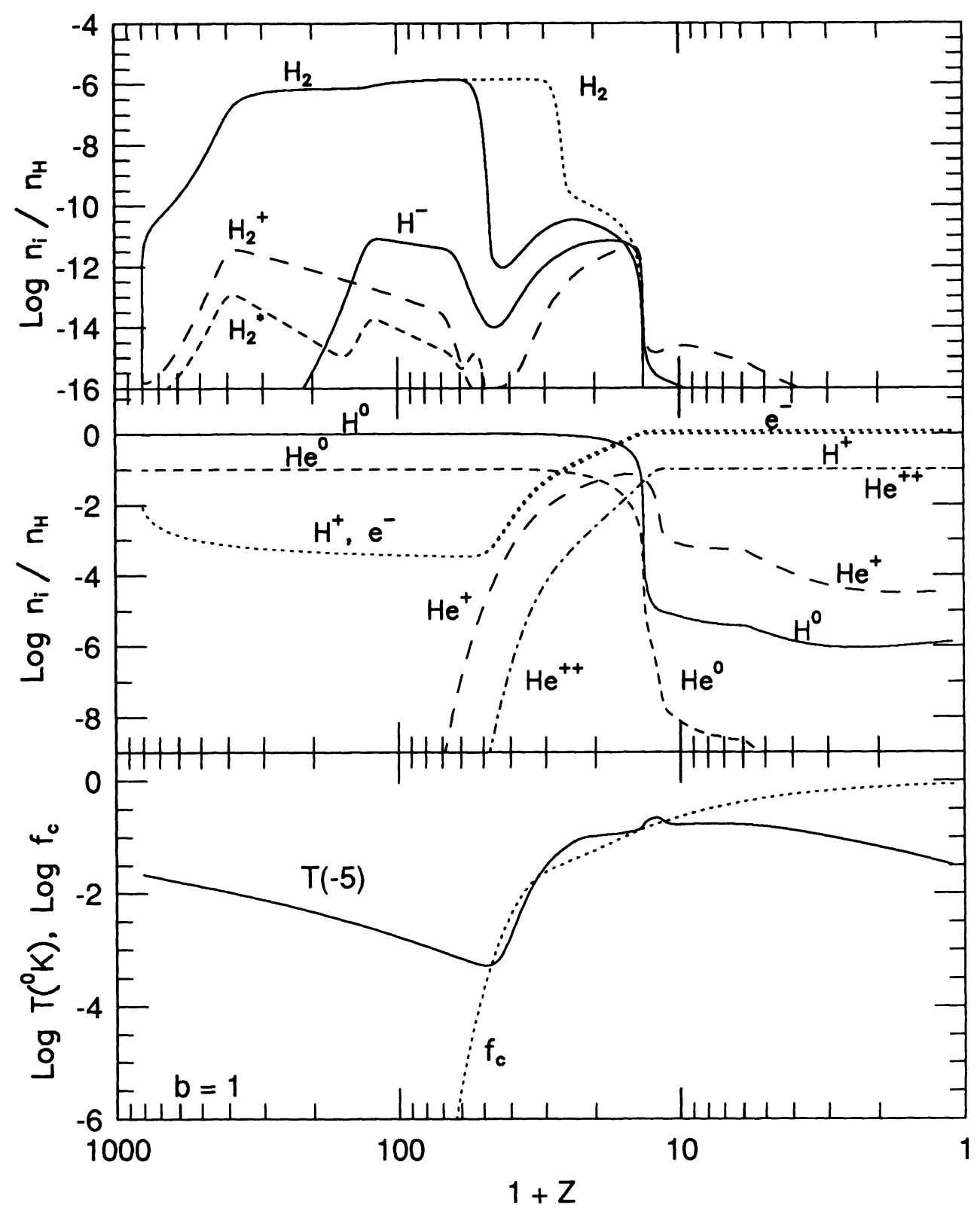

Figure 1. IGM temperature $\mathrm{T}\left({ }^{\circ} \mathrm{K}\right)\left(\times 10^{-5}\right)$, collapsed baryon fraction $f_{c}$, and species concentration by number relative to $\mathrm{H}, n_{i} / n_{H}$, as labeled for bias $b=1$. Solid curve for $\mathrm{H}_{2}$ assumes unshielded photodissociation; dashed $\mathrm{H}_{2}$ curve neglects photodissociation. 
formation. We have numerically solved the hydrodynamical conservation equations, along with the rate equations for nonequilibrium ionization, recombination, molecule formation and dissociation, and the equation of radiative transfer, in detail for steady-state, planar shocks of velocity in the range $20 \leq v_{s} \leq 400 \mathrm{~km} \mathrm{~s}^{-1}$ for a range of conditions appropriate for shocks in the IGM and inside protogalaxies [22-29]. (Calculations for shocks in the IGM were also reported by [20].) We include the same species as in the calculations for Figure 1. Figure 2 shows results for shocks inside protogalaxies, with $v_{s}=300 \mathrm{~km} \mathrm{~s}^{-1}$ such as would be produced by the typical gravitationally-induced motions within a protogalaxy, in a gas of preshock density $n_{H, 1}=0.1$. The shocked gas generally cools faster than it can recombine and, as a result, is able to form an $\mathrm{H}_{2}$ concentration as high as $10^{-3}$ or higher via the $\mathrm{H}^{-}$and $\mathrm{H}_{2}^{+}$processes, thanks to the enhanced nonequilibrium ionization at $10^{4} \mathrm{~K}$. With such an $\mathrm{H}_{2}$ concentration, the gas cools rapidly by $\mathrm{H}_{2}$ rotational-vibrational line excitation to $T \sim 10^{2}-10^{3} \mathrm{~K}$, well below the canonical final temperature of $10^{4} \mathrm{~K}$ for a molecule-free gas without metals. This cooling below $10^{4} \mathrm{~K}$ significantly lowers the characteristic gravitationally unstable fragment mass for such shocks, relative to the value if the gas cooling stops at $10^{4} \mathrm{~K}$.

This presents a problem for the suggestion that globular clusters formed within protogalaxies by gravitational instability in the compressed gas resulting from radiative shocks with velocities of the order of the virial velocity of the protogalaxy or from thermal instability in gas at the virial temperature $[35,36]$. Under such circumstances, gas which cools to $10^{4} \mathrm{~K}$ and, thereafter, remains at $10^{4} \mathrm{~K}$ for a time longer than its internal free-fall time will lead naturally to gravitational instability with a characteristic mass comparable to those of globular clusters. If, instead, the gas forms enough $\mathrm{H}_{2}$ to cool too rapidly below $10^{4} \mathrm{~K}$, however, the model fails. Our shock calculations have led to a new version of the model, in which a strong enough UV or soft X-ray source $\left(L \gtrsim 10^{45} \mathrm{erg} \mathrm{s}^{-1}\right)$, such as a quasar or early-type stars, is required to be present within the protogalaxy during the globular cluster formation epoch to suppress $\mathrm{H}_{2}$ formation in the shocks [26].

Figure 3 shows the effect of adding to the shock in Figure 2 a preshock magnetic field of strength $B_{1}=1 \mu G$, oriented parallel to the shock front [29]. Flux-freezing and the nearly isobaric compression of the radiatively cooling postshock gas together cause magnetic pressure to dominate downstream before the gas reaches $\sim 10^{4} \mathrm{~K}$. This halts the compression and makes the photon-to-atom ratio higher in the temperature plateau at $10^{4} \mathrm{~K}$ than in the nonmagnetic case. The latter encourages the photodestruction of $\mathrm{H}_{2}$ and makes it easier to suppress $\mathrm{H}_{2}$ formation and cooling with a given radiation flux level than in the nonmagnetic case, thereby lowering the threshold level required for the globular cluster formation model (e.g. $L \sim 10^{44} \mathrm{erg} \mathrm{s}^{-1}$ or less, for $\left.B_{1} \gtrsim 1.6 n_{H, 1}^{1 / 2} \mu G\right)$. 


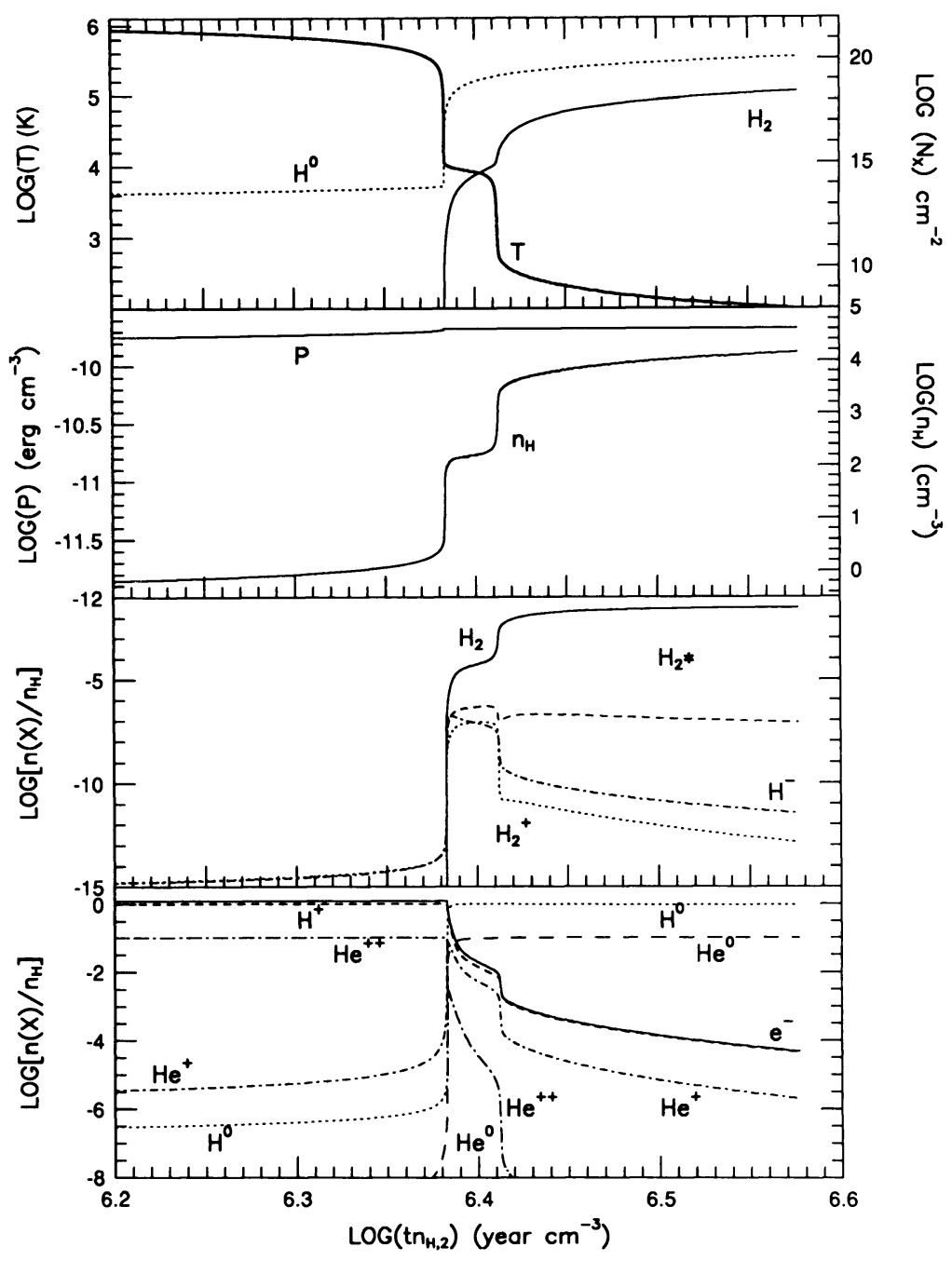

Figure 2. Postshock flow quantities versus $t n_{H, 2}\left(\right.$ years $\left.\mathrm{cm}^{-3}\right)$ for $v_{s}=300 \mathrm{~km} \mathrm{~s}$ and $n_{H, 1}=0.1 \mathrm{~cm}^{-3}$, where $n_{H, 2}$ is postshock $\mathrm{H}$ atom density and $\mathrm{t}$ is time since fluid element was shocked. Horizontal coordinate also corresponds to $\mathrm{N}_{\mathrm{H}} \mathrm{cm}^{-2}$, total $\mathrm{H}$ column density between fluid element and shock, at time $t$, where $N_{H}=$ $n_{H, 1} v_{s} t=\left(n_{H, 1} / n_{H, 2}\right) v_{s} t n_{H, 2}$, as long as we take $\log _{10} N_{H}=\log _{10}\left(t n_{H, 2}\right)+$ 14.382. Top panel shows temperature (scale at left) and total $\mathrm{H}^{0}$ and $\mathrm{H}_{2}$ column densities between fluid element and shock (scale at right) as labeled. Second panel shows gas pressure (scale at left) and $\mathrm{H}$ atom density (scale at right) as labeled. Third and fourth panels show concentrations $n_{i} / n_{H}$ as labeled. 


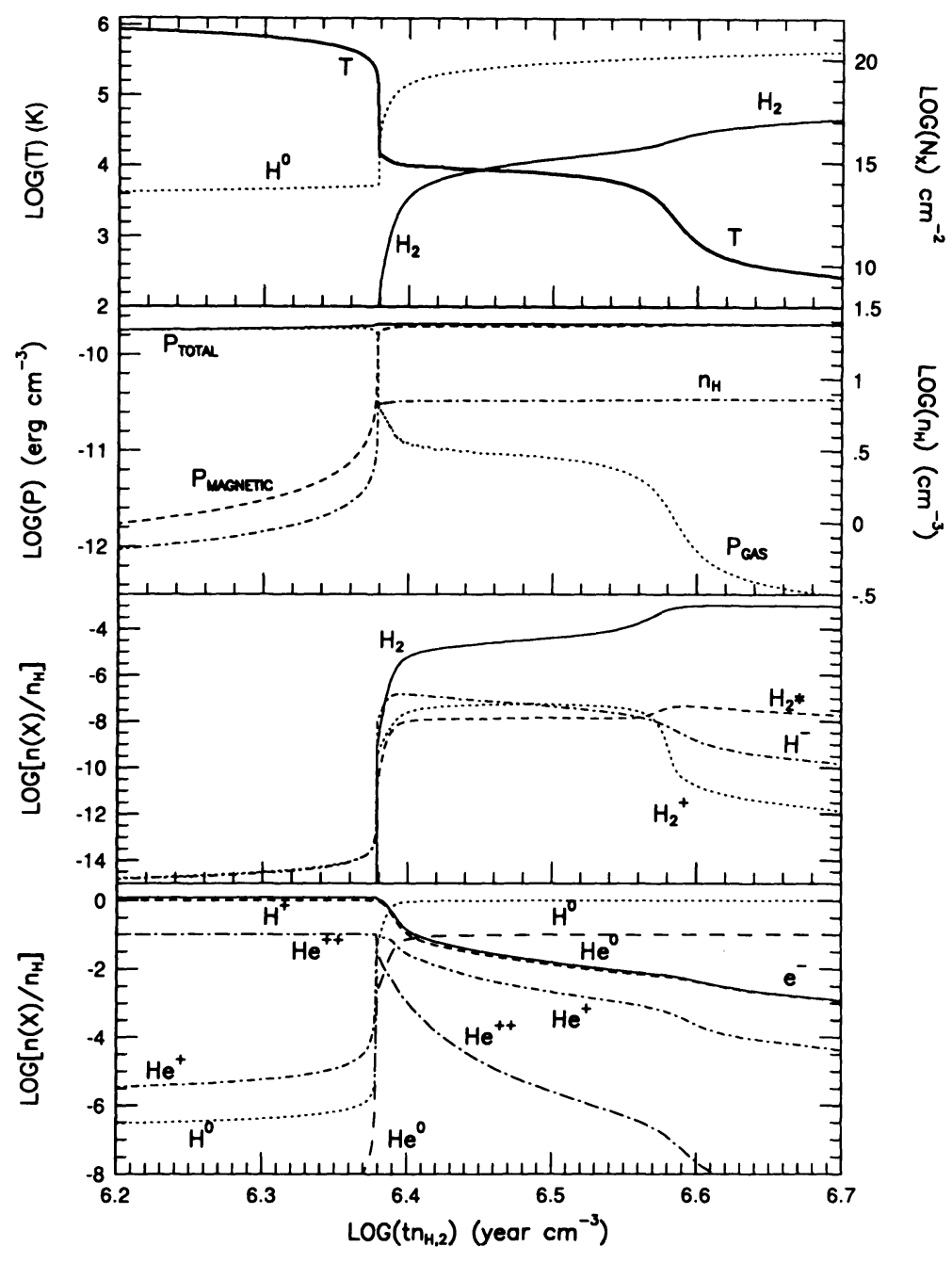

Figure 3. Same as Figure 2, but magnetized case with $B_{1}=1 \mu G$. All panels are the same as in Figure 2 except the second, where gas, magnetic and total pressure are plotted, along with $\mathrm{H}$ atom density. 
Acknowledgments. I am especially grateful to my collaborators M. Giroux, H. Kang, and A. Clocchiatti. This work was supported in part by Robert A. Welch Foundation Grant F-1115, Texas Advanced Research Program Grant 4132, NASA Grants NAGW-2399, NGT-50316, and NGT-50519. Our numerical computations were performed on the University of Texas Center for High Performance Computing Cray Y/MP.

\section{References}

1. Lepp, S. and Shull, J. M. (1984) 'Molecules in the Early Universe', Ap. J., 280, 465-469.

2. Latter, W. B. and Black, J. H. (1991) 'Molecular Hydrogen Formation By Excited Atom Radiative Association', Ap. J., 372, 161-166

3. Couchman, H.M.P. (1985) 'Reheating of the Intergalactic Medium at $z>10$ ', M.N.R.A.S., 214, 137-159.

4. Giroux, M. L. and Shapiro, P. R. (1992) 'The Reionization of the Intergalactic Medium', Ap. J., to be submitted.

5. Peebles, P.J.E. and Dicke, R. H. (1968) 'Origin of the Globular Star Clusters', Ap. J., 154, 891-908.

6. Hirasawa, T., Aizu, K., and Taketani, M. (1969) 'Formation of Galaxies from Hydrogen Gas', Progr. Theor. Phys., 41, 835-838.

7. Hirasawa, T. (1969) 'Formation of Protogalaxies and Molecular Processes in Hydrogen Gas', Progr. Theor. Phys., 42, 523-543.

8. Matsuda, T., Sato, H., and Takeda, H. (1969) 'Cooling of Pre-Galactic Gas Clouds by Hydrogen Molecule', Progr. Theor. Phys., 42, 219-232.

9. Yoneyama, T. (1972) 'On the Fragmentation of a Contracting Hydrogen Cloud in an Expanding Universe', Pub. Astr. Soc. Japan, 24, 87-98.

10. Hutchins, J. B. (1976) 'The Thermal Effects of $\mathrm{H}_{2}$ Molecules in Rotating and Collapsing Spheroidal Gas Clouds', Ap. J., 205, 103-121.

11. Silk, J. (1977) 'On the Fragmentation of Cosmic Gas Clouds. I. The Formation of Galaxies and the First Generation of Stars', Ap. J., 211, 638-648.

12. Carlberg, R. G. (1981) 'An Estimate of the Mass of Zero Metal Stars', M.N.R.A.S., 197, 1021-1029.

13. Palla, F., Salpeter, E. E., and Stahler, S. W. (1983) 'Primordial Star Formation: The Role of Molecular Hydrogen', Ap. J., 271, 632-641.

14. Izotov, Yu. I and Kolesnik, I. G. (1984) 'Kinetics of $\mathrm{H}_{2}$ Formation in the Primordial Gas', Soviet Astr., 28, No. 1, 15-21. 
15. Murray, S. D. and Lin, D.N.C. (1989) 'The Fragmentation of Proto-Globular Clusters. I. Thermal Instabilities', Ap. J., 339, 933-942.

16. Murray, S. D. and Lin, D.N.C. (1990) 'On the Fragmentation of Protogalactic Clouds', Ap. J., 363, 50-56.

17. Struck-Marcell, C. (1982) 'Gas Cloud Collisions in Protogalaxies: I. Numerical Simulations', Ap. J., 259, 116-126.

18. Struck-Marcell, C. (1982) 'Star Formation in Protogalactic Gas Cloud Collisions', Ap. J., 259, 127-132.

19. Shapiro, P. R. (1986) 'Extragalactic Gas at High Redshift: A Chronograph of Nonlinear Departures from Hubble Flow', in B. F. Madore and R. B. Tully (eds.), Galaxy Distances and Deviation from Universal Expansion, Reidel, Dordrecht, pp. 203-213.

20. MacLow, M. M. and Shull, J. M. (1986) 'Molecular Processes and Gravitational Collapse in Intergalactic Shocks', Ap. J., 302, 585-589.

21. Shapiro, P. R. and Kang, H. (1987), 'Hydrogen Molecules and the Radiative Cooling of Pregalactic Shocks', in G. R. Knapp and J. Kormendy (eds.), IAU Symposium 117: Dark Matter in the Universe, Reidel, Dordrecht, p. 365.

22. Shapiro, P. R. and Kang, H. (1987) 'Hydrogen Molecules and the Radiative Cooling of Pregalactic Shocks', Ap. J., 318, 32-65.

23. Shapiro, P. R. and Kang, H. (1987) 'Hydrogen Molecules and the Radiative Cooling of Pregalactic Shocks II: Low Velocity Shocks at High Redshift', Rev. Mexicana Astron. Astrof., 24, 58-65.

24. Shapiro, P. R., Giroux, M. L., and Kang, H. (1987) 'New Results in the Theory of the Intergalactic Medium at High Redshift', in J. Bergeron, D. Kunth, B. Rocca-Volmerange, and J. Tran Thanh Van (eds.), High Redshift and Primeval Galaxies, Editions Frontières, pp. 501-515.

25. Shapiro, P. R. and Kang, H. (1990) 'Radiative Shocks and Nonequilibrium Chemistry in the Early Universe: Galaxy and Primordial Star Formation', in R. Capuzzo-Dolcetta et al. (eds.), Physical Processes in Fragmentation and Star Formation, Kluwer Academic, Dordrecht, pp. 55-70.

26. Kang, H., Shapiro, P. R., Fall, S. M., and Rees, M. J. (1990) 'Radiative Shocks Inside Protogalaxies and the Origin of Globular Clusters', Ap. J., 363, 488-498.

27. Kang, H. and Shapiro, P. R. (1992) 'Radiative Shocks and Hydrogen Molecules in Pregalactic Gas: The Effects of Postshock Radiation', Ap. J., 386, 432-451.

28. Shapiro, P. R., Clocchiatti, A., and Kang, H. (1991) 'Magnetic Fields and Radiative Shocks in Protogalaxies and the Origin of Globular Clusters', in K. Janes (ed.), The Formation and Evolution of Star Clusters, (Astronomical Society of the Pacific Conference Series, Vol. 13), pp. 176-179. 
29. Shapiro, P. R., Clocchiatti, A., and Kang, H. (1992), 'Magnetic Fields and Radiative Shocks in Protogalaxies and the Origin of Globular Clusters', Ap. J., 387, in press.

30. Dalgarno, A. and Lepp, S. (1987) 'Chemistry in the Early Universe', in M. S. Vardya and S. P. Tarafdar (eds.), I.A.U. Symposium 120: Astrochemistry, Kluwer Academic, Dordrecht, pp. 109-120.

31. Walker, T. P., Steigman, G., Schramm, D. N., Olive, K. A., and Kang, H. S. (1991) 'Primordial Nucleosynthesis Redux', Ap. J., 376, 51-69.

32. Saslaw, W. C. and Zipoy, D. (1967) 'Molecular Hydrogen in Pre-galactic Gas Clouds', Nature, 216, 976-978.

33. Shapiro, P. R., Giroux, M. L., and Babul, A. (1991), 'The Evolving Intergalatic Medium: The Uncollapsed Baryon Fraction in a Cold Dark Matter Universe', in S. S. Holt, C. L. Bennett, and V. Trimble (eds.), After The First Three Minutes (American Institute of Physics Conference Proceedings No. 222), pp. 347-351.

34. Shapiro, P. R., Giroux, M. L., and Babul, A. (1992) 'The Evolving Intergalatic Medium: The Uncollapsed Baryon Fraction in a Cold Dark Matter Universe', Ap. J., to be submitted.

35. Fall, S. M. and Rees, M. J. (1985) 'A Theory for the Origin of Globular Clusters', Ap. J., 298, 18-26.

36. Fall, S. M. and Rees, M. J. (1988) 'The Origin of Globular Clusters', in J. E. Grindlay and A.G.D. Philip (eds.), I.A.U. Symposium 126: Globular Cluster Systems in Galaxies, Reidel, Dordecht, pp. 323-332.

\section{QUESTIONS AND ANSWERS}

L.Blitz: I thought that the lowest temperature you could cool pure $H_{2}$ gas was $\sim 500 \mathrm{~K}$ which is the temperature of the lowest quadrupole $\mathrm{H}_{2}$ rotational transition. Your curves level off at temperatures a. little more than $100 \mathrm{~K}$. Where does the other factor of $4-5$ in the temperature come from?

P.R.Shapiro: Although it is true that the lowest quadrupole rotational transition for $\mathrm{H}_{2}$ corresponds to an excitation energy of $510 \mathrm{~K}$ (in temperature units), this only means that the collisional excitation rate for this transition is exponentially cut-off at temperatures below $510 \mathrm{~K}$. Hence, while the rate is reduced by this exponential $\mathrm{T}$ dependence, it is not zero. Another words, the high energy tail of the $\mathrm{H}$ atom Maxwellian velocity distribution still has enough energy per atom to excite the $\mathrm{H}_{2}$ transition. In addition, in our post shock gas, as the temperature drops below $500 \mathrm{~K}$, the $\mathrm{H}_{2}$ concentration continues to increase, so this offsets the exponential fall-off of the $\mathrm{T}$ dependence of the excitation cooling rate per $\mathrm{H}_{2}$ molecule. 